\section{Molecular Diagnosis in an Argentinean Mitochondrial Disorders Cohort}

\author{
Nerina Martinez ${ }^{1,2}$ (D), Julieta Rosales ${ }^{1}$, Nancy Medina ${ }^{1}$, Josefina \\ Perez-Maturo',2, Valeria Salinas ${ }^{1,2}$, Lucia Zavala ${ }^{1}$, Patricia Vega', \\ Sergio Rodríguez-Quiroga ${ }^{1,3}$, Dolores González-Morón' \\ and Marcelo A. Kauffman ${ }^{1,2}$
}

Journal of Inborn Errors of Metabolism \& Screening 2021, Volume 9: e20200020 DOI: https://doi.org/10.1590/2326-4594JIEMS-2020-0020

\begin{abstract}
Mitochondrial diseases are multisystemic disorders characterized by an impairment of the mitochondrial respiratory chain. Diagnosis requires an approach that involves a high index of suspicion, molecular techniques and a careful selection of the tissue to be studied. Our goal was to develop and implement local strategies for diagnosing mitochondrial disorders, by standardizing procedures of molecular biology and nucleic acid sequencing. A prospective, analytical, observational study was conducted in a cohort of, a total of 82 patients with suspected mitochondrial disorder who were treated at our hospital between May 2008 and June 2019. We developed molecular diagnostic tools that included classical monogenic techniques and Next Generation Sequencing. We characterized the neurological and extra neurological manifestations noted in our cohort. Following the proposed algorithm, we obtained a molecular diagnostic performance of 54\%, identifying mutations in 44 patients. mtDNA mutations were identified in 34 patients. Structural rearrangements in mitochondrial genome were found in 3 and 7 in nuclear genes, respectively. Our results confirm the utility of the proposed algorithm and the molecular tools used, as evidenced by a high diagnostic performance. This is of great value to a more efficient and comprehensive medical care of patients and families affected by mitochondrial disorders.
\end{abstract}

\title{
Keywords
}

Mitochondrial diseases, Molecular diagnostic, Algorithm.

\section{Introduction}

Mitochondrial diseases are multisystemic disorders characterized by an impairment of the mitochondrial respiratory chain (RC). The deficiency of the mitochondrial RC result in a lack of ATP production, the energy provider for each cell through oxidative phosphorylation (OXPHOS). Most of the proteins required for the correct functioning of mitochondria are encoded by nuclear genes (nDNA), but 13 subunits of RC complexes are encoded by the mitochondrial genome (mtDNA) [1]. There are particular features of mtDNA that distinguish it from the nuclear genome: (a) each cell has hundreds to thousands of copies of mtDNA; (b) It is inherited only from the mother; (c) there could be a mixture of mtDNA populations in different proportions, where some harbor mutant alleles and some contain wild type sequences, a phenomenon called heteroplasmy [2]. Determining the level of heteroplasmy is important in mitochondrial disease diagnosis [3]. Due to this, the diagnosis of mitochondriopathies is challenging.

\footnotetext{
${ }^{1}$ Centro Universitario de Neurología "José María Ramos Mejía", Facultad de Medicina, División Neurología, Hospital JM Ramos Mejía, Consultorio y Laboratorio de Neurogenética, UBA, Argentina.

${ }^{2}$ Universidad Austral-CONICET, Facultad de Ciencias Biomédicas, Instituto de Investigaciones en Medicina Traslacional, Programa de Medicina de Precisión y Genómica Clínica, Pilar, Argentina.

${ }^{3}$ Centro Universitario de Neurologia "Jose Maria Ramos Mejia", Facultad de Medicina, División Neurología, Área de Trastornos del Movimiento, Hospital JM Ramos Mejía, UBA, Argentina.
}

Received November 13, 2020, and in revised form February 4, 2021. Accepted for publication February 9, 2021.

\section{Corresponding Author:}

Marcelo A. Kauffman, Facultad de Medicina, Centro Universitario de Neurología "José María Ramos Mejía” y División Neurología, Hospital JM Ramos Mejía, Consultorio y Laboratorio de Neurogenética, UBA, Argentina.

Email: marcelokauffman@gmail.com 
Frequently organs with highest needs of energy such as the brain, heart, eyes, and muscles are affected the most. Furthermore, mitochondrial disorders, can manifest at any age. Adding to the complexity of these disorders is poor genotype phenotype correlations, i.e. The same genotype can cause different phenotypes (m.3243A > G causes MELAS, CPEO) and the same phenotype can be caused by many different genotypes (Leigh syndrome has more than 75 single gene etiologies). [4].

Finding the pathogenic variants by molecular genetic testing confirms the diagnosis, and provides the ability to deliver genetic counseling. However, the genetic etiology remains to be identified in most patients suspected with mitochondrial disorders. NextGeneration Sequencing (NGS) has improved the diagnostic efficiency and facilitated the molecular routine diagnosis of such diseases in terms of cost and time incurred. [5,6].

Our goal was to develop and implement local strategies for diagnosing mitochondrial disorders, by standardizing procedures of molecular biology and nucleic acid sequencing.

\section{Materials and Methods}

\section{Patients: Diagnostic Procedure}

A prospective, observational, and descriptive cohort study of patients with clinical suspicion of mitochondrial disorder was conducted. We included patients that have been evaluated from May 2008 to June 2019 at the Neurogenetic Unit from the Neurology Division of the J.M. Ramos Mejía Hospital in Buenos Aires, Argentina. All the patients, before they participated in the study, provided informed consent through a form approved by the Ethics Committee of the institution. In all of the patients, we used a structured clinical interview in order to register demographic characteristics, family history, and the main clinical features of their complaint.

A diagnostic approach based on three stages was followed in each patient (Figure 1). Briefly, it consisted of successive stages of characterization and the leading of diagnostic procedures aimed at the individualization of the etiopathogenic molecular defect.

The first step was to determine the probability that the patient has a mitochondrial disease. Wolf's scale was selected to stratify this probability through the sum of the signs and symptoms together with the findings in complementary studies allow obtaining a total score on the modified Wolf scale. The higher this score, the greater the likelihood of mitochondrial dysfunction as an etiology of the assisted disorder. [7]. In a second stage, the selection of the molecular tests to be performed in each patient was made. Despite the frequent symptomatic and genetic heterogeneity of mitochondrial disease, there are well-recognized syndromes (MELAS, MERRF, CPEO, Leigh, SANDOS, etc.) that allow the selection and targeting of molecular tests for diagnosis of mitochondrial disorders. Accordingly, point mutations and /or structural alterations in the mtDNA or candidate nuclear genes

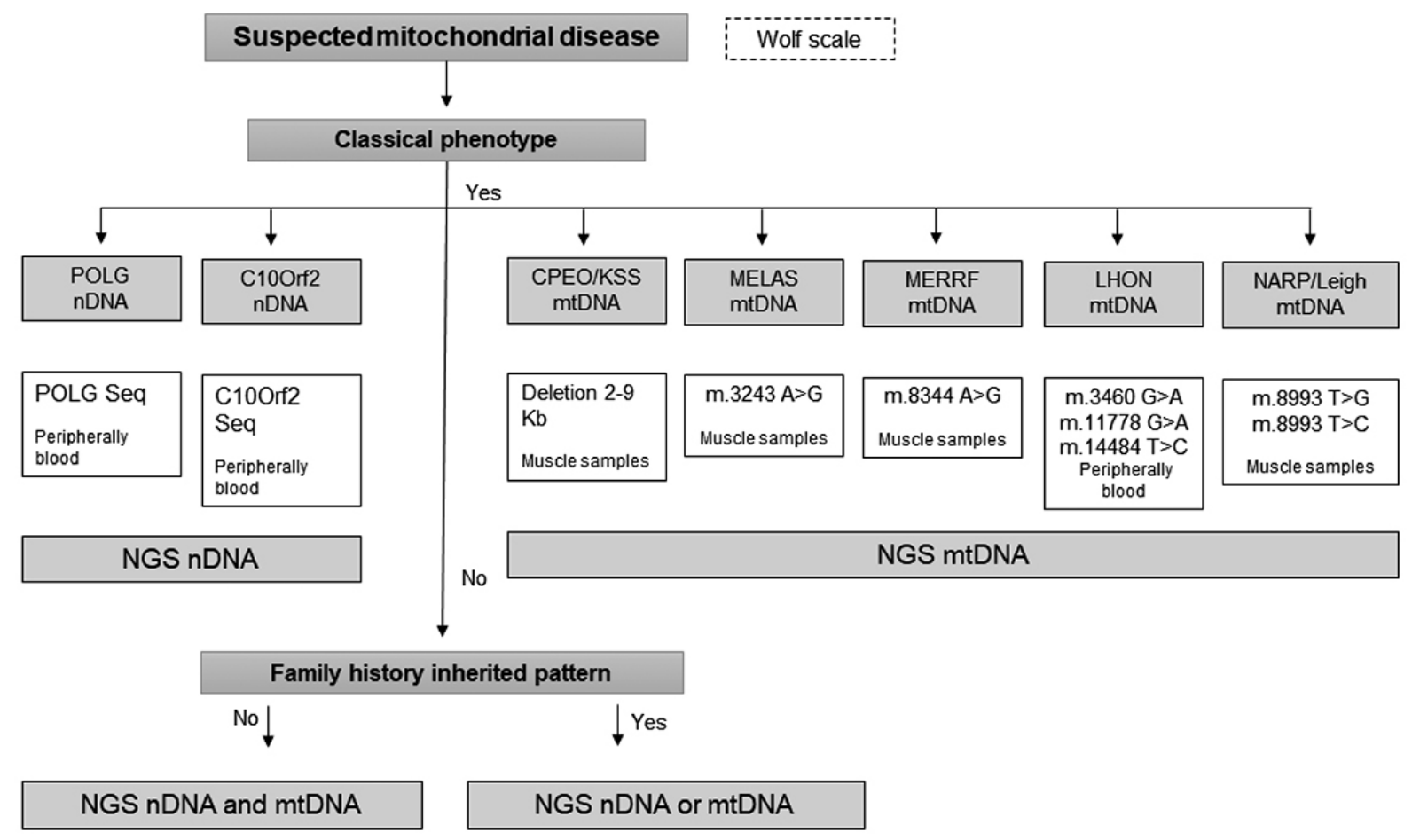

Figure 1. Clinical-molecular proposed algorithm: The first step is to determine what is the probability that the patient has a mitochondrial disease. In the second step, it is determined if it has a phenotypic presentation corresponding to a classic syndrome or not. If the patient presents a classic phenotype, the search for point mutations and / or structural alterations in the mtDNA or the sequencing of candidate nuclear genes such as C10Orf2 or POLG, as appropriate, is performed. In the event that these studies are negative, sequencing of the complete exome and / or the mitochondrial genome is continued, as indicated by the pattern of inheritance observed in the family studied. 
such as C10orf2 or POLG were looked at in patients showing a classical phenotype, as appropriate. Those cases where the subjects presented undetermined phenotypes or nondiagnostic first tier tests were studied by means of NGS assays that included mtDNA whole genome sequencing.

\section{Genomic DNA Purification}

Genomic DNA was purified from blood from all patients and from muscle samples in case of heteroplasmic pathologies, using a commercial kit from Qiagen (Qiagen N.V.) following manufacturer instructions. This was kept anonymized until further processing.

\section{Sanger Sequencing of CLassics Phenotypes}

Flanking regions of mtDNA, exons 1 and 2 of the C10orf2 gene and exons 1 to 23 of the POLG gene were amplified by PCR to find point mutations, (primers and conditions are available upon request). The PCR products were sequenced through Sanger sequencing by capillary electrophoresis (ABI BigDye terminator).

\section{Next Generation Sequencing of mtDNA}

For Next-generation sequencing assays, we first amplified fragments of about $8,5 \mathrm{~Kb}$ overlapping the whole mtDNA of patients employing two long-range PCR reactions using Ranger DNA Polymerase and Mix (Bioline, Inc). PCR products were checked by agarose electrophoresis and thereafter purified through a commercial column-based purification system. Indexed sequencing libraries of the PCR products were constructed using the Illumina Nextera XT DNA Library Prep Kit (Illumina, San Diego, CA, USA) according to manufacturer's protocol. NGS sequencing runs were made in an Illumina MiSeq (Illumina, San Diego, CA, USA) system generating $2 \times 150$-bp reads. A median sequence coverage of 5,000 $\times$ was obtained. Reads were aligned to the human mitochondrion, complete genome (NC_012920.1) using the Burrows-Wheeler alignment tool (BWA-MEM) [8] and variant calls were generated using GATK haplotype caller. Integrative genomics viewer (IGV v.2.4) [9] was used to examine the variant quality and read counts of the target amplicons [10].

\section{Results}

\section{Cohort}

Between May 2008 and June 2019, a total of 82 patients with suspected mitochondrial disorders were included in this study. Following the proposed algorithm, the causal defect was found in 44 patients, obtaining in this way a diagnostic yield of 54\% (Table 1). Our cohort presented a similar representation of sex (19 women and $25 \mathrm{men}$ ). The average age of onset of symptoms was 23 years, with a mínimum of 6 months and a máximum of 64 years. All diagnosed patients presented phenotype compatible with a classical mitochondrial syndrome, the most frequent were LHON (20 patients), followed by CPEO (11 patients), MELAS (9 patients), MERRF (3 patients) and Leigh (1 patient). Thereof 17 were sporadic, while 23 had a family history (Maternal inheritance: 17 and Autosomal dominant: 6).

\section{Clinical Manifestations}

The most frequent neurological manifestation was muscle weakness in limbs, followed by bilateral optic neuropathy, ptosisophthalmoparesis, encephalopathy, isolated ptosis, epilepsy, ataxia, stroke-like episodes, neuropathy, dysphagia with other bulbar symptoms, myoclonus, pigmentary retinopathy, and much less frequently headache and dystonia. Extra-neurological manifestations included bilateral sensorineural deafness, arrhythmias, glucose intolerance, cardiomyopathies, lipomas, and short stature.

\section{Molecular Diagnostic Performance}

Point mutations in the mtDNA were individualized in 34 patients. Structural alterations in the mitochondrial genome in 3 patients and mutations in nuclear genes in 7 patients (Table 2).

\section{Case Presentation}

Two clinical cases are described illustrative of the clinical pleomorphism of mitochondrial diseases, their diagnostic difficulty and the need for molecular tools for their diagnosis.

Case 1: A 34 year old female patient, which began at the age of 17 with bilateral palpebral ptosis and slowly progressive ophthalmoparesis. At the age of 30 , she added proximal 4-limb weakness and speech-swallowing difficulties. His family history was relevant due to the presence of bilateral ptosis in his mother and sister. On physical examination, she showed bilateral $2 / 3$ palpebral ptosis, moderate ophthalmoparesis, nasal voice and bilateral hearing loss. Mild proximal 4-limb weakness (Kendall 3-4/5), preserved sensitivity and normorreflexia were evident too. Paraclinical tests also confirmed the presence of sensorineural hearing loss, glucose intolerance, cardiac supraventricular conduction disorder, increased creatine kinase and lactic acid in the blood. The phenotype was interpreted as CPEO Plus. Muscle biopsy showed a high percentage of Cox deficient RRF. Sanger sequencing of mtDNA found the m.3243A $>\mathrm{G}$ in peripheral blood and in muscle, thus a high level of heteroplasmy was inferred (Figure 2).

Case 2: A 26 year old man, without personal or familial antecedents started at the age of 8 with learning difficulties. 12 years later, he added trunk ataxia with right thrust, behavioral disorders, and exercise intolerance. At the age of 25 , he presented dysarthria and homonymous right hemianopia focal motor seizure in his upper right limb. A Brain MRI evidenced a left parietotemporal-occipital cortico-subcortical lesion with diffusion restriction, which remitted spontaneously and completely after one month, being interpreted as a "stroke like" 
Table 1. Detail of the patients in whom a molecular defect was identified and their phenotypic presentation

\begin{tabular}{|c|c|c|c|c|c|c|}
\hline $\begin{array}{l}\text { Patient } \\
\text { number }\end{array}$ & Gender & $\begin{array}{l}\text { Age of onset } \\
\text { (years) }\end{array}$ & Clinical & $\begin{array}{c}\text { Family history } \\
\text { inherited pattern }\end{array}$ & Clinical diagnosis & Gene Sequencing method \\
\hline 1 & $\mathrm{~F}$ & 44 & $\begin{array}{l}\text { Epilepsy, myopathy, ptosis, stroke-like episodes, } \\
\text { bilateral sensorineural deafness, RRF }\end{array}$ & Sporadic & MELAS (NC_012920: m.3243 A>G) & Sanger Sequencing \\
\hline 2 & M & 35 & Myopathy, RRF & Sporadic & MERRF (NC_012920: m.8344 A>G) & Sanger Sequencing \\
\hline $3^{* 2}$ & $\mathrm{~F}$ & 11 & Epilepsy, myoclonus & Maternal inheritance & MERRF (NC_012920: m.8344 A>G) & Sanger Sequencing \\
\hline 4 & $\mathrm{~F}$ & 40 & Ptosis-ophthalmoparesis & Autosomal dominant & $\begin{array}{l}\text { CPEO (NM_021830.4 (C10orf2) } \\
\text { p.Arg334GIn (c.1001G>A)) }\end{array}$ & Sanger Sequencing \\
\hline 5 & $\mathrm{~F}$ & 4 & $\begin{array}{l}\text { Ptosis-ophthalmoparesis, myopathy, ataxia, } \\
\text { bilateral sensorineural deafness, arrhythmias, RRF }\end{array}$ & Sporadic & $\begin{array}{l}\text { KSS (NC_012920: } \\
\text { m.8482_13460del4977) }\end{array}$ & Sanger Sequencing \\
\hline 6 & M & 19 & $\begin{array}{l}\text { Ptosis-ophthalmoparesis, sensorineural deafness, } \\
\text { cardiomyopathies, myopathy, RRF }\end{array}$ & Sporadic & $\begin{array}{l}\text { CPEO (NC_012920: } \\
\text { m.8482_13460del4977) }\end{array}$ & Sanger Sequencing \\
\hline 7 & M & 15 & $\begin{array}{l}\text { Subacute bilateral } \\
\text { Central scotoma } \\
\text { Severe visual loss }\end{array}$ & Sporadic & LHON (NC_012920: m.3460 G>A) & Sanger Sequencing \\
\hline 8 & $\mathrm{~F}$ & 21 & $\begin{array}{l}\text { Subacute bilateral } \\
\text { Central scotoma } \\
\text { Severe visual loss }\end{array}$ & Sporadic & LHON (NC_012920: m.11778 G>A) & Sanger Sequencing \\
\hline 9 & $\mathrm{~F}$ & 22 & $\begin{array}{l}\text { Subacute Bilateral } \\
\text { Temporal scotoma } \\
\text { Severe visual loss }\end{array}$ & Maternal inheritance & LHON (NC_012920: m.11778 G>A) & Sanger Sequencing \\
\hline 10 & M & 18 & $\begin{array}{l}\text { Subacute Bilateral } \\
\text { Severe visual loss } \\
\text { Central scotoma }\end{array}$ & Sporadic & LHON (NC_012920: m.11778 G>A) & Sanger Sequencing \\
\hline 11 & M & 40 & $\begin{array}{l}\text { Bilateral } \\
\text { Severe visual loss } \\
\text { Central scotoma } \\
\text { Subacute }\end{array}$ & Maternal inheritance & LHON (NC_012920: m.3460 G>A) & Sanger Sequencing \\
\hline $12 * 11$ & $\mathrm{~F}$ & 18 & $\begin{array}{l}\text { Bilateral } \\
\text { Severe visual loss } \\
\text { Subacute }\end{array}$ & Maternal inheritance & LHON (NC_012920: m.3460 G>A) & Sanger Sequencing \\
\hline $13^{* 11}$ & M & 21 & $\begin{array}{c}\text { Bilateral } \\
\text { Severe visual loss } \\
\text { Subacute } \\
\text { Cecocentral scotoma }\end{array}$ & Maternal inheritance & LHON (NC_012920: m.3460 G>A) & Sanger Sequencing \\
\hline 14 & M & 56 & $\begin{array}{l}\text { Ptosis, glucose intolerance, } \\
\text { RRF, cardiac arrhytmia }\end{array}$ & Autosomal dominant & $\begin{array}{l}\text { CPEO (NM_021830-4 (C10Orf2) } \\
\text { p.Arg303Trp (c. } 907 \text { C>T)) }\end{array}$ & Sanger Sequencing \\
\hline
\end{tabular}


Table 1. Cont.

\begin{tabular}{|c|c|c|c|c|c|c|}
\hline $\begin{array}{l}\text { Patient } \\
\text { number }\end{array}$ & Gender & $\begin{array}{l}\text { Age of onset } \\
\text { (years) }\end{array}$ & Clinical & $\begin{array}{c}\text { Family history } \\
\text { inherited pattern }\end{array}$ & Clinical diagnosis & Gene Sequencing method \\
\hline $15^{* 14}$ & $\mathrm{~F}$ & 39 & Ptosis & Autosomal dominant & $\begin{array}{l}\text { CPEO (NM_021830-4 (C10Orf2) } \\
\text { p.Arg303Trp (c. } 907 \text { C>T)) }\end{array}$ & Sanger Sequencing \\
\hline $16^{* 14}$ & $\mathrm{~F}$ & 20 & Ptosis & Autosomal dominant & $\begin{array}{l}\text { CPEO (NM_021830-4 (C100rf2) } \\
\text { p.Arg303Trp (c. } 907 \text { C>T)) }\end{array}$ & Sanger Sequencing \\
\hline 17 & M & 17 & $\begin{array}{c}\text { Bilateral } \\
\text { Severe visual loss } \\
\text { Cecocentral scotoma } \\
\text { Subacute }\end{array}$ & Maternal inheritance & LHON (NC_012920: m.11778 G>A) & Sanger Sequencing \\
\hline 18 & M & 29 & $\begin{array}{c}\text { Bilateral } \\
\text { Severe visual loss } \\
\text { Cecocentral scotoma } \\
\text { Subacute }\end{array}$ & Sporadic & LHON (NC_012920: m.11778 G>A) & Sanger Sequencing \\
\hline 19 & M & 8 & $\begin{array}{c}\text { Epilepsy, ataxia, stroke-like episodes, encephalopathy, } \\
\text { bilateral sensorineural deafness. }\end{array}$ & Sporadic & MELAS (NC_012920: m.3243 A>G) & Sanger Sequencing \\
\hline 20 & $\mathrm{~F}$ & 17 & $\begin{array}{l}\text { Ptosis-ophthalmoparesis, myopathy, neuropathy, } \\
\text { bilateral sensorineural deafness, arrhythmias, glucose } \\
\text { intolerance, dysphagia with other bulbar symptoms, RRF }\end{array}$ & Maternal inheritance & MELAS (NC_012920: m.3243 A>G) & Sanger Sequencing \\
\hline 21 & M & 0.5 & $\begin{array}{l}\text { Encephalopathy, cardiomyopathies, } \\
\text { lactic acidosis, RRF }\end{array}$ & Sporadic & Leigh (NC_012920: m.8993 T>G) & NGS \\
\hline 22 & $\mathrm{~F}$ & 4 & $\begin{array}{c}\text { Myopathy, lipomas, ataxia, bilateral optic neuropathy, } \\
\text { lactic acidosis, RRF }\end{array}$ & Sporadic & MERRF (NC_012920: m.8344 A>G) & Sanger Sequencing \\
\hline 23 & M & 16 & $\begin{array}{c}\text { Bilateral } \\
\text { Severe visual loss } \\
\text { Central scotoma } \\
\text { Subacute }\end{array}$ & Sporadic & LHON (NC_012920: m.3460 G>A) & Sanger Sequencing \\
\hline 24 & $\mathrm{~F}$ & 31 & $\begin{array}{l}\text { Headache, stroke-like episodes, } \\
\text { epilepsy, bilateral sensorineural deafness, } \\
\text { cardiomyopathies, lactic acidosis }\end{array}$ & Maternal inheritance & MELAS (NC_012920: m.3243 A>G) & Sanger Sequencing \\
\hline 25 & $\mathrm{~F}$ & 3 & Encephalopathy, lactic acidosis & Maternal inheritance & MELAS (NC_012920: m.3243 A>G) & NGS \\
\hline $26^{* 25}$ & $\mathrm{~F}$ & 10 & Glucose intolerance & Maternal inheritance & MELAS (NC_012920: m.3243 A>G) & Sanger Sequencing \\
\hline
\end{tabular}


Table 1. Cont.

\begin{tabular}{|c|c|c|c|c|c|c|}
\hline $\begin{array}{l}\text { Patient } \\
\text { number }\end{array}$ & Gender & $\begin{array}{l}\text { Age of onset } \\
\text { (years) }\end{array}$ & Clinical & $\begin{array}{c}\text { Family history } \\
\text { inherited pattern }\end{array}$ & Clinical diagnosis & Gene Sequencing method \\
\hline 27 & M & 20 & $\begin{array}{c}\text { Bilateral } \\
\text { Severe visual loss } \\
\text { Central scotoma } \\
\text { Subacute }\end{array}$ & Maternal inheritance & LHON (NC_012920: m.11778 G>A) & Sanger Sequencing \\
\hline $28 * 27$ & M & 21 & $\begin{array}{l}\text { Bilateral } \\
\text { Severe visual loss } \\
\text { Central scotoma } \\
\quad \text { Subacute }\end{array}$ & Maternal inheritance & LHON (NC_012920: m.11778 G>A) & Sanger Sequencing \\
\hline 29 & $\mathrm{~F}$ & 18 & $\begin{array}{l}\text { Ptosis, epilepsy, encephalopathy, } \\
\text { bilateral sensorineural deafness, myopathy, lactic acidosis }\end{array}$ & Maternal inheritance & MELAS (NC_012920: m.3243 A>G) & Sanger Sequencing \\
\hline 30 & $\mathrm{~F}$ & 10 & Ptosis-ophthalmoparesis, myopathy & Sporadic & $\begin{array}{l}\text { CPEO (NC_012920: } \\
\text { m.8470_13446del4977) }\end{array}$ & Sanger Sequencing \\
\hline 31 & M & 12 & Ptosis-ophthalmoparesis, myopathy, epilepsy, RRF & Sporadic & CPEO (NC_012920: m.5703 G>A) & NGS \\
\hline 32 & M & 18 & $\begin{array}{l}\text { Bilateral } \\
\text { Severe visual loss } \\
\text { Central scotoma } \\
\text { Subacute }\end{array}$ & Sporadic & LHON (NC_012920: m.3460 G>A) & Sanger Sequencing \\
\hline 33 & M & 30 & $\begin{array}{l}\text { Bilateral } \\
\text { Severe visual loss } \\
\text { Centra scotoma } \\
\text { Subacute }\end{array}$ & Sporadic & LHON (NC_012920: m.11778 G>A) & Sanger Sequencing \\
\hline 34 & M & 19 & $\begin{array}{c}\text { Bilateral } \\
\text { Severe visual loss } \\
\text { Cecocentral scotoma } \\
\text { Subacute }\end{array}$ & Sporadic & LHON (NC_012920: m.11778 G>A) & Sanger Sequencing \\
\hline 35 & M & 20 & $\begin{array}{l}\text { Bilateral } \\
\text { Severe visual loss } \\
\text { Central scotoma } \\
\text { Subacute }\end{array}$ & Maternal inheritance & LHON (NC_012920: m.11778 G>A) & Sanger Sequencing \\
\hline 36 & M & 25 & $\begin{array}{l}\text { Bilateral } \\
\text { Severe visual loss } \\
\text { Central scotoma } \\
\text { Subacute }\end{array}$ & Maternal inheritance & LHON (NC_012920: m.11778 G>A) & Sanger Sequencing \\
\hline 37 & M & 26 & $\begin{array}{l}\text { Bilateral } \\
\text { Severe visual loss } \\
\text { Central scotoma } \\
\text { Subacute }\end{array}$ & Maternal inheritance & LHON (NC_012920: m.14484 T>C) & Sanger Sequencing \\
\hline 38 & M & 64 & Ptosis-ophthalmoparesis, myopathy, lactic acidosis & Autosomal dominant & $\begin{array}{l}\text { CPEO (NM_021830-4 (C100rf2) } \\
\text { p.Phe478Cys (c.1433 T>C) }\end{array}$ & Sanger Sequencing \\
\hline
\end{tabular}


Table 1. Cont.

\begin{tabular}{|c|c|c|c|c|c|c|}
\hline $\begin{array}{l}\text { Patient } \\
\text { number }\end{array}$ & Gender & $\begin{array}{c}\text { Age of onset } \\
\text { (years) }\end{array}$ & Clinical & $\begin{array}{c}\text { Family history } \\
\text { inherited pattern }\end{array}$ & Clinical diagnosis & Gene Sequencing method \\
\hline 39 & M & - & $\begin{array}{c}\text { Bilateral } \\
\text { Severe visual loss } \\
\text { Central scotoma } \\
\text { Subacute }\end{array}$ & Sporadic & LHON (NC_012920: m.11778 G>A) & Sanger Sequencing \\
\hline 40 & $\mathrm{~F}$ & - & Ptosis-ophthalmoparesis, bulbar symptoms, RRF & Sporadic & $\begin{array}{l}\text { CPEO (NM_021830-4 (C10Orf2) } \\
\text { p.Phe478Cys (c.1433 T>C) }\end{array}$ & Sanger Sequencing \\
\hline 41 & $\mathrm{~F}$ & 15 & Ptosis-ophthalmoparesis, bulbar symptoms, RRF & Autosomal dominant & $\begin{array}{l}\text { CPEO (NM_021830-4 (C100rf2) } \\
\text { p.Phe478Cys (c.1433 T>C) }\end{array}$ & Sanger Sequencing \\
\hline 42 & M & 36 & $\begin{array}{l}\text { Headache, bilateral sensorineural deafness, } \\
\text { lactic acidosis }\end{array}$ & Sporadic & MELAS (NC_012920: m.3243 A>G) & Sanger Sequencing \\
\hline 43 & M & - & $\begin{array}{c}\text { Bilateral } \\
\text { Severe visual loss } \\
\text { Central scotoma } \\
\text { Subacute }\end{array}$ & Sporadic & LHON (NC_012920: m.3460 G>A) & Sanger Sequencing \\
\hline 44 & $\mathrm{~F}$ & 38 & $\begin{array}{l}\text { Intelectual Disability, } \\
\text { Miopathy, Lactic acidosis }\end{array}$ & Maternal inheritance & MELAS (NC_012920: m.3243 A>G) & Sanger Sequencing \\
\hline
\end{tabular}

CPEO: Chronic progressive external ophthalmoplegia; KSS: Kearns-Sayre syndrome; LHON: Leber hereditary optic neuropathy; MELAS: Mitochondrial encephalomyopathy, lactic acidosis, and stroke-like episodes; MERRF: Myoclonic Epilepsy Associated with Ragged Red Fibers; RRF: Ragged Red Fibers; * Related.

Table 2: Frequency of molecular defects in our cohort

\begin{tabular}{|c|c|c|}
\hline Phenotype & Mutation & $\mathbf{N}^{\circ}$ of patients \\
\hline & $m t D N A$ & 37 \\
\hline MELAS & NC_012920: m.3243 A>G & 9 \\
\hline \multirow[t]{2}{*}{ MERRF } & NC_012920: $m .8344$ A>G & 3 \\
\hline & NC_012920: m.3460 G>A & 7 \\
\hline \multirow[t]{3}{*}{ LHON } & NC_012920: m.11778 G>A & 12 \\
\hline & NC_012920: m.14484 T>C & 1 \\
\hline & NC_012920: m.5703 G>A & 1 \\
\hline \multirow[t]{2}{*}{ CPEO } & NC_012920: m.8470_13446del4977 & 1 \\
\hline & NC_012920: m.8482_13460del4977 & 2 \\
\hline \multirow[t]{3}{*}{ LEIGH } & NC_012920: $\mathrm{m} .8993 \mathrm{~T}>\mathrm{G}$ & 1 \\
\hline & nDNA & 7 \\
\hline & NM_021830-4 (C10Orf2) p.Phe478Cys & 3 \\
\hline \multirow[t]{2}{*}{ CPEO } & NM_021830-4 (C10Orf2) p.Arg303Trp & 3 \\
\hline & NM_021830.4 (C10Orf2) p.Arg334Gln & 1 \\
\hline
\end{tabular}


A

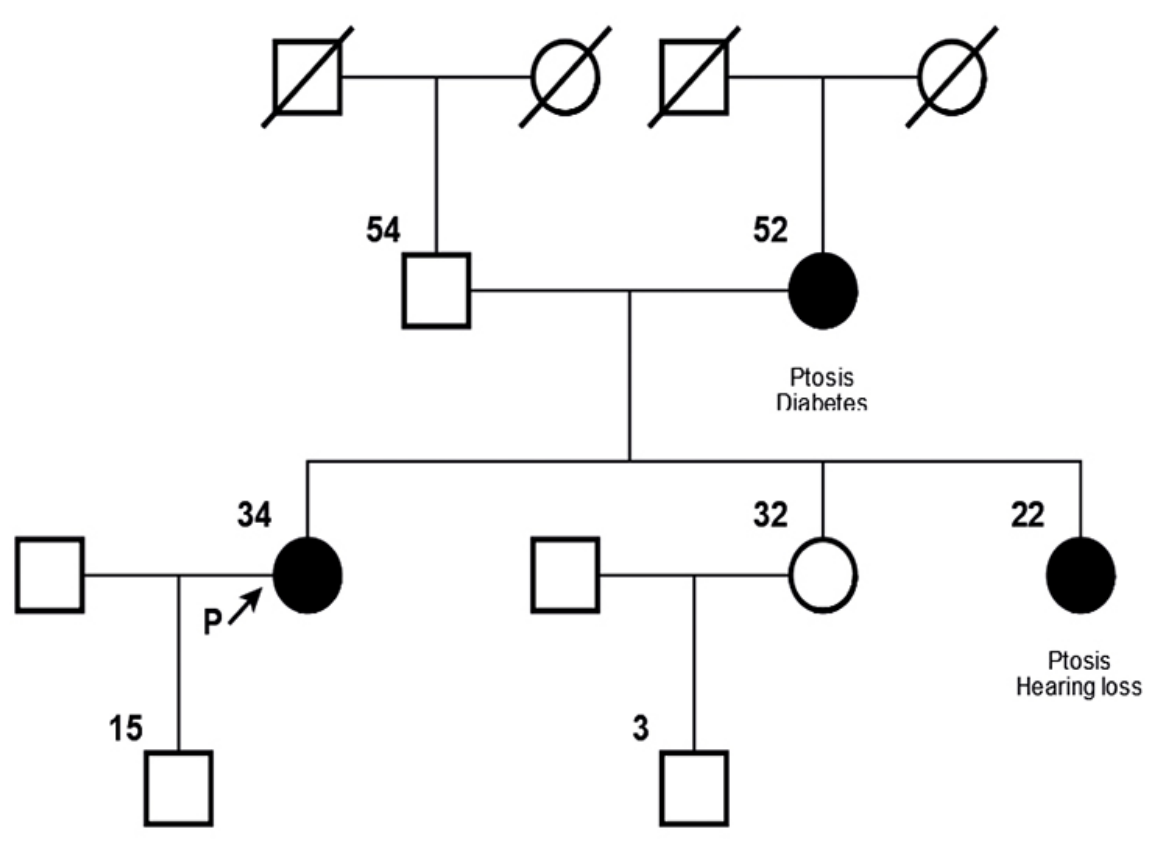

B

\section{Blood sample}

m. 3243 A $>G$

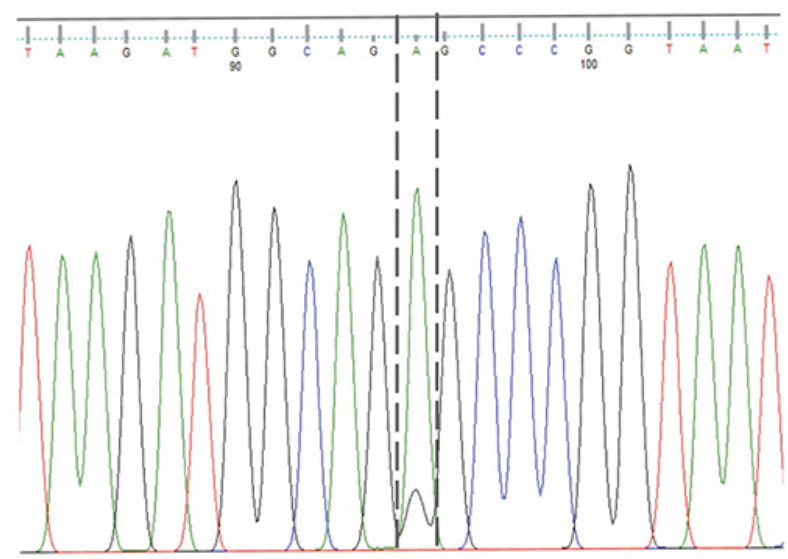

C Muscle sample

m. $3243 \mathrm{~A}>\mathrm{G}$

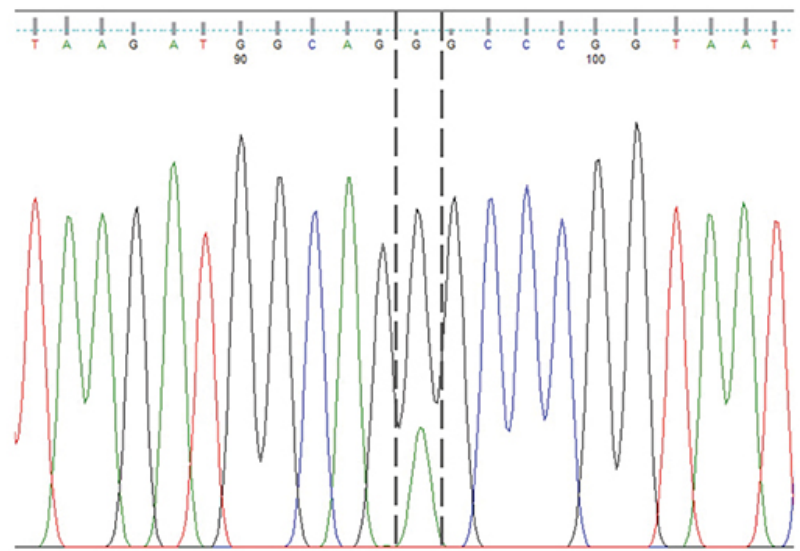

Figure 2. (a) Pedigree Chart. Arrow indicates index case. Numbers over figures corresponded to current age/age at onset. (b) Sanger Sequencing in blood sample showing identified variants in the patient. (c) Sanger Sequencing in muscle sample showing identified variants in the patient. 
episode. On physical examination, he presented MiniMental State Examination of 23/30, bilateral hearing loss and appendicular dysmetria in 4 members. The paraclinical exams also confirmed the presence of neurosensorial hearing loss and increased lactic acid in blood and fluid cerebrospinal. The phenotype was interpreted as MELAS. A muscle biopsy was performed, which showed a high percentage of Cox deficient RRF. Sequencing of the mtDNA by Sanger method also revealed the m.3243A>G mutation in muscle. (Figure 3).

A

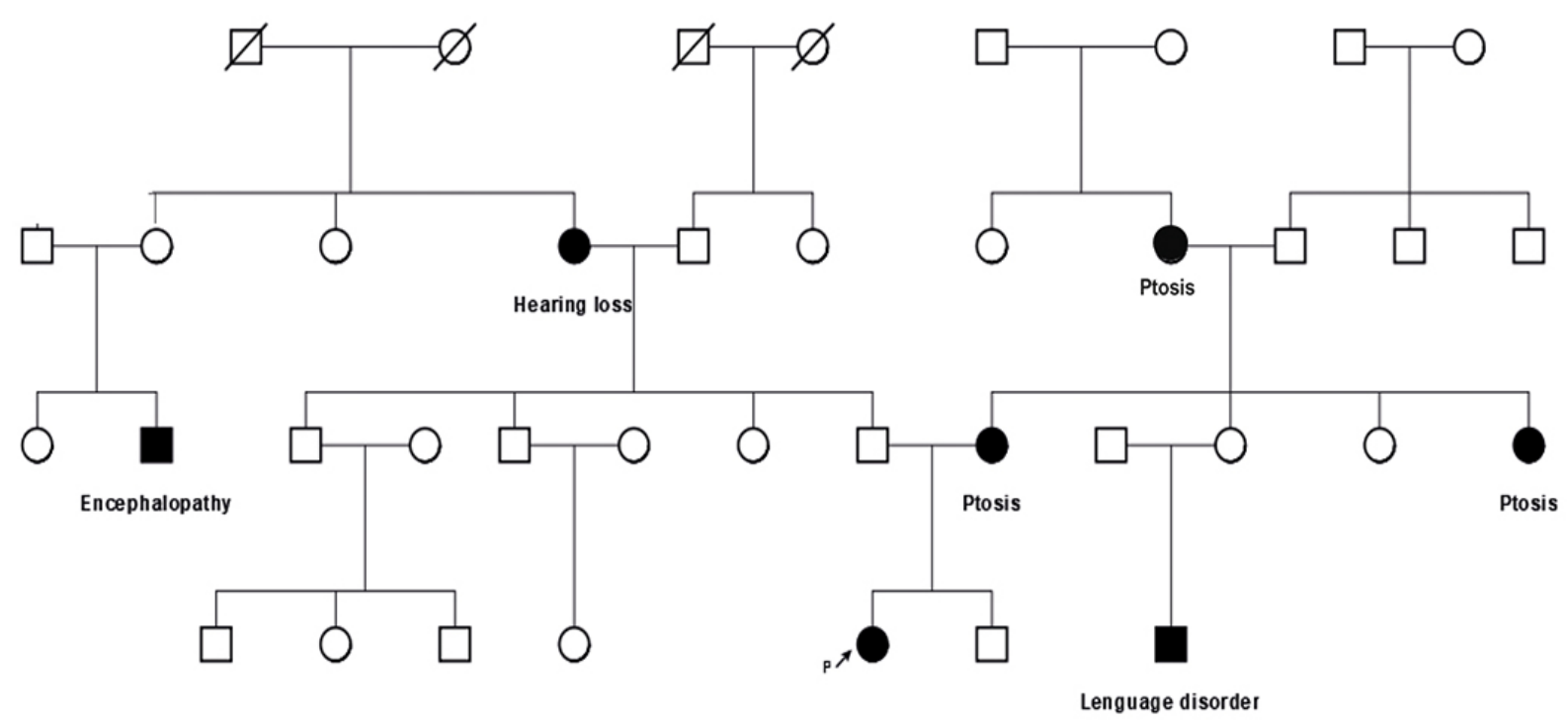

B

\section{Muscle sample}

m. $3243 A>G$

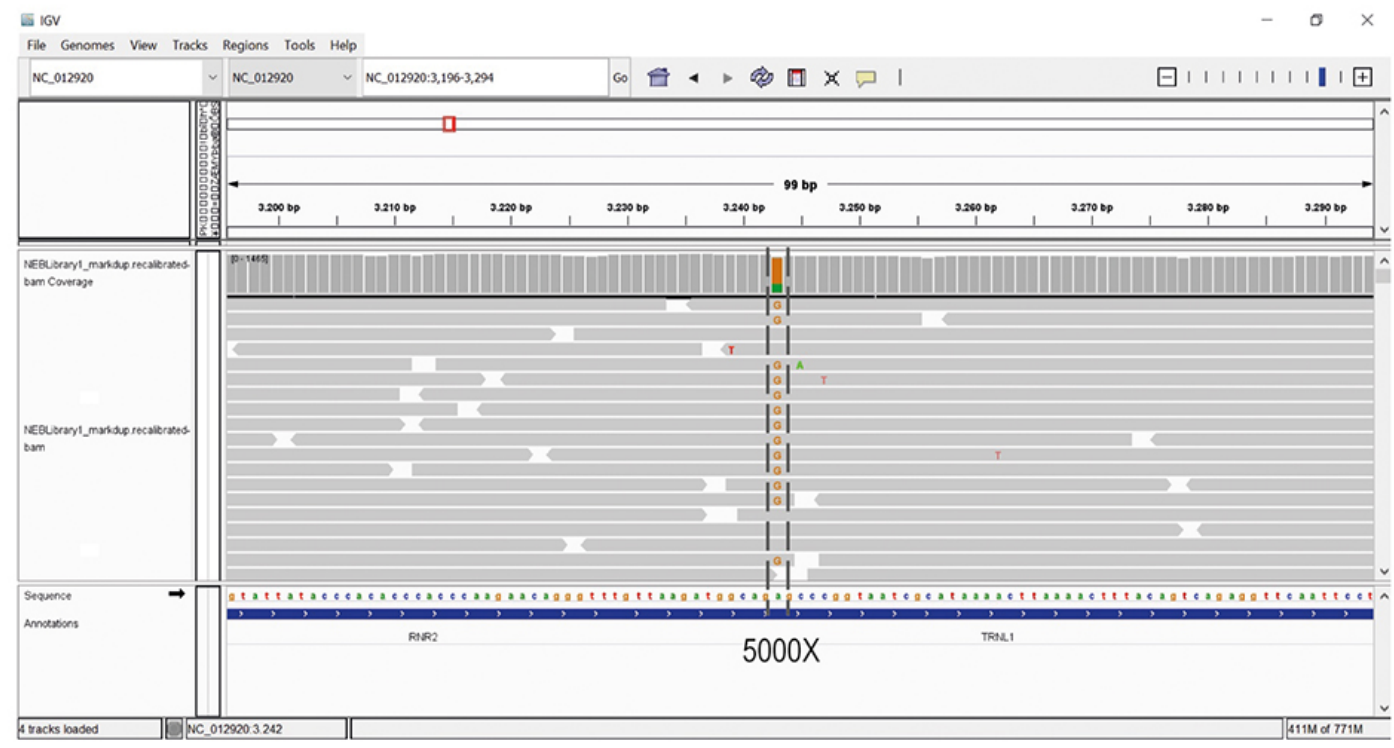

Figure 3. (a) Pedigree Chart. Arrow indicates index case (b) Alignment showing identified variants in the patient with a median sequence coverage of $5,000 \times$. 


\section{Discussion}

In the present work, a population of Argentina patients with mitochondrial disorders was characterized, implementing our algorithm that aims to order the diagnostic approach, starting with a probability score for the mitochondrial etiology of the disorder, continuing with a syndromic characterization and ending with the molecular diagnosis. As far as we know, this is the largest series reported in Argentina that includes clinical and molecular data, obtained through the own development of different methodologies that incorporate $\mathrm{nDNA}$ and $\mathrm{mtDNA}$ sequencing technologies.

Different articles published over a whole decade revealed a molecular dichotomy between adults and children, in which children frequently carry autosomal recessive disorders due to nDNA defects, while mtDNA defects appear more frequently in adults $[11,12,13,14]$. In recent years this separation has been reviewed, because nuclear mutations with Mendelian inheritance can also frequently occur in adults [15]. The main difference of our cohort in comparison with those previously reported is that we have included a majority of adults with a larger age range, with a predominance of LHON, CPEO, and MELAS as classical syndromes. Due to this, the contribution of scientific knowledge in this age group is important, including the study of mitochondrial and nuclear genes, as was done in our work.

Even though mitochondrial disorders are characterized by a marked clinical pleomorphism and a low phenotypegenotype correlation, we were able to identify the causal defect in 44 patients. Our results confirm the utility of the proposed diagnostic algorithm expressed in a high diagnostic performance (54\%), which leads to better cost-effectiveness. For example, through the NGS sequencing-based method of mtDNA, it was possible to identify a mutation in the mtDNA (m.5703G $>$ A) previously unsuspected with the phenotype. Nevertheless, we acknowledge that future prospective studies comparing this algorithm with other ones led to a more precise assessment of the methods used in our cohort.

Advances in the molecular diagnosis of mitochondrial disorders have elucidated new mechanisms in the development of clinical phenotypes that may be linked to the primary function of the mutated protein, to the secondary accumulation of deletions and point mutations of the mtDNA [16]. Moreover, the current era of expanded access to molecular diagnosis make more relevant the lack of adequate information for properly interpreting new gene variants. Series such this offers new evidence and information for the increasing number of genomic variations causing mitochondrial disorders. This opens the doors to new therapeutics aimed at preventing and repairing mtDNA damage, which could be applied at different stages of the disease.

\section{Conclussion}

We conclude that a systematized approach, such as the one we propose, allows confirming the molecular diagnosis in a high percentage of patients. This is of great value for a better understanding of the pathogenesis of neurogenetic diseases and a more efficient and comprehensive medical care for patients and their families.

\section{Ethics Approval}

This study was approved by the Institutional Ethics Committee of the Hospital JM Ramos Mejia of Buenos Aires, Argentina. All patients and parents provided written informed consent for genetic analyses and use of their anonymized data. All experiments and methods were carried out in accordance with the relevant guidelines and regulations of the Institutional Ethics Committee of the Hospital JM Ramos Mejia of Buenos Aires, Argentina. All clinical investigations have been conducted in accordance with the 1964 Helsinki Declaration and its later amendments or comparable ethical standards.

\section{Declaration of Conflict of Interest}

Nerina Martinez has received scholarship support from Argentinean National Science Council (CONICET). Marcelo Kauffman has received grant support from Ministry of Health of Buenos Aires City, Argentinean National Science Council (CONICET) and Argentinean Ministry of Science and Technology. He serves as Associate Editor of the journal Neurologia Argentina. The rest of the authors declare that they have no conflict of interest.

\section{References}

1. Alston CL, Rocha MC, Lax NZ, Turnbull DM, Taylor RW. The genetics and pathology of mitochondrial disease. $J$ Pathol. 2017;241: 336-250. doi: 10.1002/path.4809.

2. He Y, Wu J, Dressman DC, et al. Heteroplasmic mitochondrial DNA mutations in normal and tumour cells. Nature. 2010;464(7288):610-614. doi:10.1038/nature08802

3. Poulton J, Chiaratti MR, Meirelles FV, Kennedy S, Wells D, Holt IJ. Transmission of mitochondrial DNA diseases and ways to prevent them. PLoS Genet. 2010;6(8):e1001066. doi: 10.1371/journal.pgen.1001066

4. Kanungo S, Morton J, Neelakantan M, Ching K, Saeedian J, Goldstein A. Mitochondrial disorders. Ann Transl Med. 2018;6(24):475. doi: 10.21037/atm.2018.12.13

5. Lieber DS, Calvo SE, Shanahan K, et al. Targeted exome sequencing of suspected mitocondrial disorders. Neurology. 2013;80:1762-1770. doi: 10.1212/WNL.0b013e3182918c40

6. Legati A, Reyes A, Nasca A, et al. New genes and pathomechanisms in mitochondrial disorders unraveled by NGS technologies. Biochim Biophys Acta. 1857;2016:132635. doi: 10.1016/j.bbabio.2016.02.022 
7. Wolf NI, Smeitink JA. Mitochondrial disorders: a proposal for consensus diagnostic criteria in infants and children. Neurology. 2002; 59,1402-1405. doi: 10.1212/01. wnl.0000031795.91814.d8

8. Li H. Aligning sequence reads, clone sequences and assembly contigs with BWA-MEM. 2013; arXiv:1303.3997.

9. Robinson JT, Thorvaldsdóttir H, Winckler W, et al. Integrative genomics viewer. Nature Biotechnology. 2011;29(1), 24-26. doi: 10.1038/nbt.1754

10. Ramos A, Santos C, Alvarez L, Nogues R, Aluja MP Human mitochondrial DNA complete amplification and sequencing: a new validated primer set that prevents nuclear DNA sequences of mitochondrial origin co-amplification. Electrophoresis. 2009;30(9):1587-1593. doi: 10.1002/ elps.200800601

11. Wortmann SB, Koolen DA, Smeitink JA, van den Heuvel L, Rodenburg RJ. Whole exome sequencing of suspected mitochondrial patients in clinical practice. Inherit Metab Dis. 2015;38:437-443. doi: 10.1007/s10545-015-9823-y
12. Leonard JV, Schapira AH. Mitochondrial respiratory chain disorders I: Mitochondrial DNA defects. Lancet. 2000;355:299-304. doi: 10.1016/s0140-6736(99)05225-3

13. Leonard JV, Schapira AH. Mitochondrial respiratory chain disorders II: Neurodegenerative disorders and nuclear gene defects. Lancet. 2000;355:389-394. doi: 10.1016/s01406736(99)05226-5

14. Plutino M, Chaussenot A, Rouzier C, et al. Targeted next generation sequencing with an extended gene panel does not impact variant detection in mitochondrial diseases. BMC Med Genet. 2018;19:57. doi: 10.1186/s12881-0180568-y

15. Chinnery PF. Mitochondrial disease in adults: What's old and what's new? EMBO Mol Med. 2015;7:1503-1512. doi: 10.15252/emmm.201505079

16. Thompson K, Collier JJ, Glasgow RIC, et al. Recent advances in understanding the molecular genetic basis of mitochondrial disease. J Inherit Metab Dis. 2020;43(1):3650. doi: 10.1002/jimd.12104 